\title{
Current Trends and Recent Advances in Diagnosis, Therapy, and Prevention of Hepatocellular Carcinoma
}

\author{
Chun-Hsiang Wang ${ }^{\&} *$, Keh-Cherng Wey ${ }^{\star}$, Lein-Ray Mo, Kuo-Kwan Chang, \\ Ruey-Chang Lin, Jen-Juan Kuo
}

\begin{abstract}
Hepatocellular carcinoma (HCC) has been one of the most fatal malignant tumors worldwide and its associated morbidity and mortality remain of significant concern. Based on in-depth reviews of serological diagnosis of HCC, in addition to AFP, there are other biomarkers: Lens culinaris agglutinin-reactive AFP (AFP-L3), descarboxyprothrombin (DCP), tyrosine kinase with Ig and eprdermal growth factor (EGF) homology domains 2 (TIE2)-espressing monocytes (TEMs), glypican-3 (GPC3), Golgi protein 73 (GP73), interleukin-6 (IL-6), and squamous cell carcinoma antigen (SCCA) have been proposed as biomarkers for the early detection of HCC. The diagnosis of HCC is primarily based on noninvasive standard imaging methods, such as ultrasound (US), dynamic multiphasic multidetector-row CT (MDCT) and magnetic resonance imaging (MRI). Some experts advocate gadolinium diethyl-enetriamine pentaacetic acid (Gd-EOB-DTPA) MRI and contrast-enhanced US as the promising imaging madalities of choice. With regard to recent advancements in tissue markers, many cuting-edge technologies using genome-wide DNA microarrays, qRT-PCR, and proteomic and inmunostaining studies have been implemented in an attempt to identify markers for early diagnosis of HCC. Only less than half of HCC patients at initial diagnosis are at an early stage treatable with curative options: local ablation, surgical resection, or liver transplant. Transarterial chemoembolization (TACE) is considered the standard of care with palliation for intermediate stage HCC. Recent innovative procedures using drug-eluting-beads and radioembolization using Yttrium-90 may exhibit beneficial effects in HCC treatment. During the past few years, several molecular targeted agents have been evaluated in clinical trials in advanced HCC. Sorafenib is currently the only approved systemic treatment for HCC. It has been approved for the therapy of asymptomatic HCC patients with well-preserved liver function who are not candidates for potentially curative treatments, such as surgical resection or liver transplantation. In the USA, Europe and particularly Japan, hepatitis $C$ virus (HCV) related HCC accounts for most liver cancer, as compared with Asia-Pacific regions, where hepatitis B virus (HBV) may play a more important role in HCC development. HBV vaccination, while a vaccine is not yet available against $\mathrm{HCV}$, has been recognized as a best primary prevention method for HBV-related HCC, although in patients already infected with $\mathrm{HBV}$ or $\mathrm{HCV}$, secondary prevention with antiviral therapy is still a reasonable strategy. In addition to $\mathrm{HBV}$ and $\mathrm{HCV}$, attention should be paid to other relevant $\mathrm{HCC}$ risk factors, including nonalcoholic fatty liver disease due to obesity and diabetes, heavy alcohol consumption, and prolonged aflatoxin exposure. Interestingly, coffee and vitamin $\mathrm{K} 2$ have been proven to provide protective effects against HCC. Regarding tertiary prevention of $\mathrm{HCC}$ recurrence after surgical resection, addition of antiviral treatment has proven to be a rational strategy.
\end{abstract}

Keywords: Hepatocellular carcinoma - hepatitis B virus - hepatitis C virus - transarterial chemoembolization

Asian Pac J Cancer Prev, 16 (9), 3595-3604

\section{Introduction}

Hepatocellular carcinoma (HCC) is the fifth most common cancer in men worldwide $(523,000$ cases per year, $7.9 \%$ of all cancers) and the seventh in women (226,000 cases per year, $6.5 \%$ of all cancers) (El-Serag, 2012). Patients with chronic liver disease are at the highest risk for developing this tumor.

The globally uneven distribution of HCC is mostly due to the prevalence of hepatotropic viruses, mainly hepatitis $\mathrm{B}$ virus (HBV) and hepatitis $\mathrm{C}$ virus (HCV), varying geographically. The burden of $\mathrm{HCC}$ is believed to be the highest in Eastern Asia, sub-Saharan Africa, where HBV infection is endemic. On the contrary, in United States, Europe, and particularly Japan, HCV infection is prevalent, and is the major risk factor for acquiring HCC in these areas (Parkin et al., 2005)

Recent major advances in diagnostic technologies 
not only enhance accuracy, feasibility and safety but also provide additional information to help in patients' management. This article will cover several recent studies from a critical viewpoint.

The spectrum of HCC treatment options in conventional clinical practice includes surgical resection, liver transplantation, local ablation, and systemic cytotoxic or targeted therapy. Assessing all of the possible treatment strategies, there is still relevant unmet goal in current therapeutic approaches, which indeed need significant improvement. Current research trends with respect to future perspectives on HCC treatment will be comprehensively reviewed.

The issue of HCC prevention will be addressed in terms of three stages, including primary, secdary, and tertiary prevetion with different approaches on the basis of relevant data from recent published advanced studies.

\section{Diagnosis}

Patients at risk of $\mathrm{HCC}$, which include cirrhosis due to $\mathrm{HBV}, \mathrm{HCV}$, alcohol, genetic hemochromatosis, non-alcoholic steatohepatitis, primary biliary cirrhosis, alpha1-antitrypsin deficiency, other causes of cirrhosis, and HBV carriers without cirrhosis, should be monitored every six months with alpha-fetoprotein (AFP) and liver ultrasound (US) examination aimed at the early detection of HCC (NCCN., 2013).

There is an array of diagnosis criteria of HCC, including the detection of the index tumor, intrahepatic tumor staging, and assessing extrahepatic metastasis.

The tests used to diagnose HCC usually include cross-sectional diagnostic imaging, serological diagnosis, and histological diagnosis. Cross-sectional diagnostic imaging, including US, computed tomography (CT), and magnetic resonance imaging (MRI), is crucial not only in the diagnosis but also in assessing tumor staging and therapeutic response of HCC. Nowadays, CT and MRI are mostly used for further assessing unusual cases with US screening and to determine magnitude of disease.

The most commonly used routine periodic surveillance tests of individuals with HCC are AFP and hepatic US, which must be performed half a year in terms of doubling time of tumor progression (Franca et al., 2004). There is a potential complementary role for US and AFP (Lok et al., 2010). The potential benefit of combining US with AFP for detection of early stage HCC was reported in the previously mentioned meta-analysis by Singal et al (2009). Therefore, the combination of AFP and US is indeed an indispensable method for the surveillance of HCC in patients with cirrhosis (Andea et al., 2009).

As a frontline HCC screening tool, AFP is the most frequently used tumor marker worldwide. Besides, AFP has a role in the diagnosis of $\mathrm{HCC}$, because a hepatic tumor with AFP greater than a cutoff value of $200 \mathrm{ng} / \mathrm{mL}$ in cirrhotic patients has a very high positive predictive value for HCC (Trevisani et al., 2001; Tateishi et al., 2008). In addition, using AFP to detect cancer has assisted to screen for HCC and save medical resources. Nevertheless, It should also be emphasized that not all HCC has elevated AFP level. Also, AFP might be abnormal in patients having chronic liver disease without HCC. Even other malignant tumors were reported to have elevated AFP level (Di Bisceglie et al., 2005). In addition to AFP, there are several tumor markers that are found to be elevated in patients with HCC and may aid in the diagnosis of HCC, such as AFP-L3 fraction which is elevated in HCC and improves the specificity of AFP (Sterling et al., 2007), and descarboxyprothrombin (DCP), also known as prothrombin Induced by vitamin $\mathrm{K}$ Ab- sence II (PIVKA II) (Tsai et al., 1990; Koike et al., 2001; Marrero et al., 2003). However, it has been recognized that the use of DCP have been focused on a diagnostic role rather than surveillance.

Recently published results from Matsubara et al. (2013) reported that Tyrosine kinase with Ig and eprdermal growth factor (EGF) homology domains 2 (TIE2)expressing monocytes (TEMs) was superior to AFP and DCP in the diagnosis of HCC. Several recent studies (Capurro et al., 2003; Giannelli et al., 2005; Marrero et al., 2005; Hsia et al., 2007) further have shown that Glypican-3 (GPC3), Golgi protein 73 (GP73), interleukin-6 (IL-6), and squamous cell carcinoma antigen (SCCA) may help diagnose HCC. Another silmilar novel markers include highly upregulated in liver cancer (HULC) long noncoding RNAs (lncRNAs), FK506 binding protein 11 (FKBP11), Dickkopf-1 (Dkk-1), and MicroRNAs (miRNAs), which have the potential to be promising and early biomarkers for HCC (Lin et al., 2013; Qi, 2013; Xie et al., 2013). However, above-mentioned new biomarkers have still to be validated before entering general use.

US is generally used for HCC surveillance in chronic liver disease worldwide, mostly because of its ease of approach, lack of radiation, and relatively inexpensive, compared to CT and MRI. HCC detection by US is relatively inaccurate, whereas $\mathrm{CT}$ or MRI have established a typical imaging profile of HCC (Yu et al., 2011). In the arterial phase, it was observed that HCC enhances more intensely than the surrounding liver. This is because the HCC mainly contains arterial blood. In the venous phase, the HCC enhances less than the surrounding liver. This is known as "washout" (Burrel et al., 2003; Forner et al., 2008). Hence, the presence of arterial uptake followed by washout is highly specific for HCC. Forner et al. (2008) used contrast-enhanced ultrasound (CEUS) and MRI to evaluate lesions smaller than $2 \mathrm{~cm}$ found on surveillance. The positive predictive value of using these two tests was $100 \%$, although the negative predictive value was only about $42 \%$. This means that if both tests are positive the lesion is always HCC. It is evident that classical HCC is mainly fed by the hepatic artery. Therefore, imaging of hepatic blood flow, including arterial and portal venous flow, is important for diagnosing liver tumors. Dynamic CT, MRI, CT during arterial portography (CTAP), and CT hepatic arteriography (CTHA) have rationally been developed to evaluate hepatic blood flow (Lee et al., 2012). Moreover, MRI with tissue-specific MR contrast media and CEUS with real-time high-spatial-resolution imaging have recently become clinically available (Joo and Choi, 2012). Dynamic MRI with gadolinium diethyl-enetriamine pentaacetic acid (Gd-EOB-DTPA), a hepatocyte-specific contrast agent, is commonly used on 
dynamic MRI because Gd-EOB-DTPA-enhanced MRI has been reported to considerably improve diagnostic accuracy of HCC and has a sensitivity of $91-93 \%$ for small HCC of less than $2 \mathrm{~cm}$ in size (Park et al., 2012; Ichikawa et al., 2014). Gd-EOB-DTPA-enhanced MRI is helpful in diagnosing benign tumors, such as hypervascular pseudotumors, focal nodular hyperplasia, and nodular lesions associated with alcohol-induced hepatitis, as well as some hypervascular malignant tumors, such as cholangiolocellular carcinoma, mixed type tumors, and metastatic liver tumors, which all required differentiation from HCC (Murakami and Tsurusaki, 2014). However, if there are still uncertainties on imaging diagnosis of HCC, biopsy may be able to resolve doubts about the diagnosis. Therefore, further clarification by final pathological results might be able to affect patient management. Despite its indispensable role of HCC diagnosis, liver biopsy still has some inevitable limitations, including tumor seeding risk and sampling error.

As a histologically diagnostic tool for HCC, International Consensus Group for Hepatocellular Neoplasia (2009) has now been implemented for pathological diagnosis; however, given the ambiguousness of morphological diagnostic criteria for high-grade dysplastic nodules versus early HCC (Roskams and Kojiro, 2010), there were some uncertainties. Because the cutting-edge technologies of tissue markers has been advancing, the above uncertain issue was solved. Distinct technologies such as genome-wide DNA microarray, qRTPCR, proteomic and inmunostaining studies have been used in an attempt to identify markers for early diagnosis of HCC (Villanueva et al., 2010). Immunohistochemistrycross-sectional diagnostic imaging markers of early HCC identified by genomic studies has therefore been developed. One of these markers is GPC3, which shows a sensitivity of $68-72 \%$ with a specificity superior to $92 \%$ (Di Tommaso et al., 2007). Moreover, combinations of different protein markers, HSP70, GPC3, and GS, in 105 hepatocellular nodules performed well with a sensitivity and a specificity of $72 \%$ and $100 \%$, respectively (Capurro et al., 2003). Dhayat et al. (2014) in their recent innovative study reported that the miR-200 family as important epigenetic regulators of epithelial-mesenchymal transition (EMT), such as ZEB-1, E-cadherin and vimentin, are able to distinguish between cirrhotic and $\mathrm{HCC}$ tissue and could serve as an early marker for cirrhosis-associated HCC.

\section{Treatment}

HCC treatment depends on the tumor stage, patient performance status and liver function reserve and requires a multidisciplinary approach. There has been general consensus of treatment guidelines derived from National Comprehensive Cancer Network (NCCN), Asian Pacific Association for the Study of the Liver (APASL), American Association for the Study of Liver (AASLD), World Gastroenterology Organisation (WGO), European Association for the Study of the Liver (EASL), and Italian Association of Study of the Liver (AISF). Manifold current treatment choices are available for HCC including curative resection, liver transplantation, radiofrequency ablation (RFA), transarterial chemoembolization (TACE), radioembolization and systemic targeted agent like sorafenib. In the real world scenario, HCC therapies are categorized as curative and palliative, which depend upon the tumor features, hepatic reserve, presence or not of extrahepatic metastasis or vacular invasion. In the clinical setting, patients should be stratified by disease stages based on most used staging system, the Barcelona Clinic Liver Cancer (BCLC) staging system (Llovet et al., 1999). The majority of HCC patients, different from other solid tumors, usually have cirrhotic liver in addition to the neoplasm, which means that their outcomes are causally connected with both tumor itself and liver cirrhosis. The BCLC staging classification, using variables related to tumor stage, liver functional status, physical status, and cancer-related symptoms, can successfully link the stage of the disease to a specific treatment strategy. In clinical practice, for optimizing the therapeutic outcome, multidisciplinary evaluation to assess hepatic reserve and comorbidity should be performed after HCC is confirmed in order to find out patients for potentially curative options, such as resectable and transplatable patients.

Surgical resection is not only considered as a preferred strategy in terms of long-term survival ( $\mathrm{Ng}$ et al., 2005; Pawlik et al., 2005), but also is the treatment of choice, if patients' general performance status and hepatic reserve allow such a surgical management (Llovet et al., 1999)

In potentially transplatable patients, they should meet the United Network for Organ Sharing (UNOS) criteria: a tumor $\leq 5 \mathrm{~cm}$ or $2-3$ tumors $\leq 3 \mathrm{~cm}$ each, no macrovascular involvement, and no extrahepatic disease. In contrast, according to the Milan criteria, liver transplantation for patients with early HCC restricted to a solitary nodule $<5$ $\mathrm{cm}$ or three nodules, each $<3 \mathrm{~cm}$ lead to an expected 4 -year overall survival of $85 \%$ and a recurrence-free survival of 93\% (Mazzaferro et al., 1996).

One of the core principles of HCC treatment is that it is essential to consider the status of chronic liver disease prior to surgical resection, focusing on preserving hepatic function that is often already damaged. A key step in the choice of therapy is the correct assessment of the functional reserve of the liver, which is often more important than the tumor staging itself. In addition, it is useful to identify whether or not HCC is a solitary tumor and there is normal portal pressure. Therefore, it's reasonable to believe that the long-term outcome of HCC treated by surgical resection remains a concern because of subsequent recurrence of tumor, which is the main cause of mortality in addition to concomitant hepatic failure.

Those who are ineligible for liver transplant or surgical resection need to be treated with local ablation, such as RFA, which is the best local treatment, compared with percutaneous ethanol injection or microwave therapy, for patients with early $\mathrm{HCC}$ and their liver function are very well maintained (Shiina et al., 2005).

TACE has been considered a palliative treatment modality because of its incomplete tumor necrosis effect in spite of repeated TACE procedure (Bruix and Sherman, 2011). Recent data indicate that the overall objective response rate to TACE was only $35 \%$, and the 2-year survival rate was $41 \%$ (Llovet and Bruix, 2003). 
Nevertheless, TACE is still recommended as a standardof-care therapy for intermediate-stage HCC, which is defined as extensive multifocal tumors without vascular invasion in patients with preserved liver function and absence of cancer-related symptoms based on treatment guidelines of the BCLC staging system (Llovet et al., 1999).

Because aggressively repeated TACE may incur increasing adverse events, current TACE treatment strategies continue to evolve to achieve better outcome and less adverse events. One of the progress is the introduction of embolic microspheres that have the ability to be coated with chemotherapeutic drugs, such as doxorubicin, via drug-eluting beads (DEB) and release them evenly and slowly in a sustained manner that might deminish side effects of chemotherapy. The positive action of chemotherapeutic agent over the bland embolic bead in TACE procedure has been evaluated in a randomized control trial, which showed that the overall response rate was better in the doxorubicin-eluting bead group compared to the flat embolization group. This implies that DEB may exert a favorable influence on better control of tumor progression without obvious adverse event than standard TACE (Malagari et al., 2010).

Advanced HCC sometimes causes vascular thrombosis within portal vein, either in the main portal trunk or the branches, hepatic vein trunk, or inferior vena cava. These conditions can be fatal, and the prognosis of patients with portal vein tumor thrombosis (PVTT) remains very grave. The current therapeutic option is radiotherapy (RT), which can produce survival benefits in patients with advanced HCC and macroscopic hepatic vascular invasion. Recent data demonstrated that RT could exhibit more improved survival than chemotherapy, particularly in patients with advanced unresectable HCC and PVTT (Nakazawa et al., 2014).

Targeted chemotherapy, as shown in Table 1, appear to be an attractive alternative to conventional systemic chemotherapy. Among these, sorafenib, a first-line therapy for $\mathrm{HCC}$, is an oral multikinase inhibitor that targets vascular endothelial growth factor (VEGF) receptors, Raf kinase, and platelet-derived growth factor receptor-b with antiangiogenic and antiproliferative effects that significantly improves time-to-tumor progression and overall survival of patients with advanced HCC and is widely used and well-accepted to treat advanced HCC in which curative therapy is not indicated (Kudo et al., 2011). Another important area of research concerning tageted therapies of HCC is the development of secondline therapeutic agents for those who do not tolerate to sorafenib or even show tumor progression Wilhelm et al., 2004). Several compounds have been developed during the past few years and are undergoing clinical trials. Among these, tivantinib is a selective oral inhibitor targeting the MET tyrosine kinase that has shown prospect in HCC treatment. Another second-line agent under development is cabozantinib, which is a dual c-MET/VEGFR-2 inhibitor. Interim analysis demonstrated progression-free survival of 4.2 months and median overall survival of 15.1 months (Cohn et al., 2012; Zhu et al., 2013).

Table 1. Targeted Therapy for Advanced HCC

\begin{tabular}{|c|c|c|}
\hline Drugs & Molecular mechanisms & Therapeutic effects \\
\hline \multicolumn{3}{|l|}{ The first-line treatments } \\
\hline Sorafenib & $\begin{array}{l}\text { raf-, vascular endothelial growth factor } \\
\text { (VEGF) receptor-, platelet-derived growth } \\
\text { factor receptor-blocking multikinase } \\
\text { tyrosine kinase inhibitor }\end{array}$ & $\begin{array}{l}\text { 1. overall survival: } 10.7 \text { months (Western } \\
\text { study); } 6.5 \text { months (Asia-Pacific study) } \\
\text { 2. time to progression: } 5.5 \text { months (Western } \\
\text { study); } 2.8 \text { months (Asia-Pacific study) }\end{array}$ \\
\hline Sunitinib & $\begin{array}{l}\text { multi-targeted receptor tyrosine kinase } \\
\text { inhibitor tyrosine kinase inhibitor }\end{array}$ & $\begin{array}{l}\text { 1.overall survival: } 10.7 \text { months } \\
\text { 2. time to progression: } 5.5 \text { months }\end{array}$ \\
\hline Brivanib & $\begin{array}{l}\text { VEGF-receptor-, fibroblast growth factor } \\
\text { receptor-blocking multikinase tyrosine } \\
\text { kinase inhibitor }\end{array}$ & overall survival: 9.5 months \\
\hline Linifanib & $\begin{array}{l}\text { PDGF/VEGF-receptor tyrosine kinase } \\
\text { inhibitor }\end{array}$ & overall survival: 9.1 months \\
\hline Lenvatinib & $\begin{array}{l}\text { multikinase tyrosine kinase inhibitor } \\
\text { inhibiting VEGFR2 and } 3\end{array}$ & Not available \\
\hline \multicolumn{3}{|l|}{ The second-line treatments } \\
\hline Brivanib-post sorafenib & $\begin{array}{l}\text { VEGF-receptor-, fibroblast growth factor } \\
\text { receptor-blocking multikinase tyrosine } \\
\text { kinase inhibitor }\end{array}$ & overall survival: 9.4 months \\
\hline Everolimus & mTOR inhibitor & overall survival: 7.6 months \\
\hline Ramucirumab & monoclonal anti-VEGFR2 antibody & Not available \\
\hline Regorafenib & raf-, VEGF-, PDGF-, Tie2-inhibitor & Not available \\
\hline Tivantinib & c-met inhibitor & Not available \\
\hline Cabozantinib & c-met inhibitor & Not available \\
\hline Refametinib & MEK-inhibitor & Not available \\
\hline
\end{tabular}


Efforts have been made to improve the outcomes of TACE with the adjuvant or concurrent use of an antiangiogenic agent. Ischemic injuries resulting from TACE can cause the upregulation of circulating vascular VEGF, which is important in HCC pathogenesis ( $\mathrm{Li}$ et al., 2004). Accordingly, some experts reported that the combined TACE and sorafenib had created promising results in recent clinical trials (Kudo et al., 2011). However, other studies did not show a clinically significant increase in survival (Lencioni et al., 2010; Park et al., 2012;). Analogously, brivanib, a selective dual inhibitor of vascular endothelial growth factor and fibroblast growth factor signaling, may also improve the effect of TACE when given as an adjuvant to TACE. However, these combination therapy did not improve overall survival rate (Kudo et al., 2014).

In recent years, there have been several attempts to investigate radioembolization, which is a procedure containing infusion of radioactive substances into the hepatic artery. The most common form of radioembolization is the use of Yttrium-90 (Y-90), a $\beta$-emitting isotope. Y-90 radioembolization is carried in glass microspheres of 20$30 \mu \mathrm{m}$ that are minimally embolic. Response rate was $42 \%$ based on the World Health Organization (WHO) criteria. The overall time to progression was 7.9 months $(95 \% \mathrm{CI}$, 6-10.3) (Hilgard et al., 2010; Salem et al., 2010).

\section{Prevention}

Up-to-date information regarding significant factors for $\mathrm{HCC}$ prevention have been fully investigated. Among these, HBV vaccine for the prevention of HBV infection is a glimpse of optimism in the field. HBV vaccination has been proven to be a factor that strongly affects the prevention of HCC development.

Baesed on reports from Chang et al., immunization of infants against HBV first began in 1984, and universal coverage was accomplished in 1986, coverage of all preschool children by 1987 and entension to older children and adults by 1990 (Chang et al., 1997). The prevalence of $\mathrm{HCC}$ among recipients of the HBV vaccine has already decreased by $70 \%$ in comparison with those in the nonvaccinated age groups (Chang et al., 2009). A nationwide vaccination program against $\mathrm{HBV}$ has reduced the $\mathrm{HBsAg}$ carrier rate in the younger population. More importantly, follow-up results have shown a significant reduction in the incidence of HCC in children. The average annual incidence of HCC in children 6-14 years of age declined from $0.70 / 100,000$ children between 1981 and 1986 to 0.57 between 1986 and 1990 , and further to $0.36 / 100,000$ between 1990 and $1994(P<0.01)$ (Hsu et al., 1988; Chen et al., 1996; Chang et al., 2000; Chang et al., 2005).

As an oncogenic virus, HBV can also cause HCC in the absence of cirrhosis by direct carcinogenic mechanisms in terms of the integration of the virus genome in the host DNA and coding for proteins such as the X protein and truncated preS-S protein which have potential transforming features (Brechot et al., 2000). Given above data, the most effective way to prevent HBV-related HCC is by vaccination, so-called "primary prvention"; however, in patients already infected with HBV, antiviral therapy is the best strategy. Antiviral thersapies, which effectively treat hepatitis $\mathrm{B}$ and $\mathrm{C}$ infection, are the options to protect chronic $\mathrm{HBV}$ and $\mathrm{HCV}$-infected patients from the occurrence of HCC (Franceschi et al., 2006; Yoshizawa et al., 2006; Lim et al., 2009). Successful treatment of chronic hepatitis B with either pegylated interferonalpha (PEG-IFN-a) or oral nucleoside and nucleotide analogues (NAs) can induce regression of fibrosis in some cases, even reduce the risk of HCC in patients with HBV infection including those with advanced liver fibrosis. Although it does not completely eliminate HCC, treatment with NAs appears to be more effective in lowering the risk of $\mathrm{HCC}$ in patients with cirrhosis (Papatheodoridis et al., 2010). Lamivudine (LAM) was shown to be effective in HCC prevention in patients with chronic hepatitis B infection. Only one RCT (Yoshizawa, 2002) suggests that LAM treatment of chronic hepatitis $\mathrm{B}$ and advanced liver disease does reduce the incidence of HCC, but with marginal significance (hazard ratio $0.49,95 \%$ CI $0.25-0.99, P=0.047)$. In another study in Japan in which patients receiving NAs were stratified by a propensity score (PS)-matched entecavir (ETV) -treated group, non- rescued LAM-treated patients, and control long-term ETV therapy to assess the chemoprevention of HCC The results showed that ETV was better than LAM in suppressing HCC, with a SVR rate of almost $90 \%$ in the ETV-treated patients at year one that was accompanied by very low rates of drug resistance $(0.8 \%)$ after a median follow-up of 3.2 years. (Hosaka et al., 2013). A recent multicenter European study involving 372 ETV-treated patients (26\% with cirrhosis) who were followed up for a median of 20 months showed that a sustained viral response (SVR) of ETV, defined as a serum HBV DNA level $<80 \mathrm{IU} / \mathrm{mL} 6$ months after end of treatment, reduced the probability of clinical events including HCC in patients with cirrhosis but not in those failing to have a virological response (Zoutendijk et al., 2013). Considering long-term outcomes, patients with chronic hepatitis B treated with ETV will develop HCC despite a viral response because ETV therapy could only suppress but not eradicate HBV. Therefore, the risk of HCC development is still present. The results of risk of HCC development following the treatment of chronic hepatitis B patients with tenofovir disoproxil fumarate (TDF) were obtained from the roll over study of registration trials in $\mathrm{HBeAg}$-positive and HBeAg-negative patients. As evidenced in this trials, TDF has also been proved to prevent the development of HCC in responders with cirrhosis, although HCC may still occur even in low risk patients (Triolo et al., 2014). Generally speaking, available evidence mentioned above suggests that the benefit of reduction of HCC risk is mainly observed in those who were successfully treated with antiviral agents.

Because it still remains a significant concern of HCC development even after antiviral therapy, efforts have been turned to vigorous HCC surveillance. In the literature, several authors have attempted to develop three risk prediction scores, namely CU-HCC score, GAGHCC score, REACH-B score, of HBV related HCC after antiviral treatment (Yuen et al., 2009; Wong et al., 2010; Yang et al., 2011). These HCC risk scores can accurately 
predict subsequent $\mathrm{HCC}$ development in both antiviral treatment-naive and experienced patients (Wong et al., 2013)

Current research trends with respect to prevention of HCV related HCC have been widely studied but remain controversial. The apparent beneficial effect of the chemoprevention by pegylated interfeon based antiviral therapy in reducing HCC was contradictory because the data from the results in recent literature were conflicting. (Mazzaferro et al., 2006; Masuzaki et al., 2010; Lok et al., 2011). Recently, it has been reported that combination therapy with direct-acting antiviral agents (DAAs), such as a nonstructural protein (NS) 3 inhibitor and a NS5A inhibitor without peg-IFN and ribavirin, was well tolerated and achieved a high prevalence of SVR (Lok et al., 2012). Ad hoc well-designed clinical trials are needed to ascertain if these DAAs possess a preventive effect in regard to the occurrence and recurrence of $\mathrm{HCC}$

Morgan et al. (2013) in their meta-analysis study showed that SVR among HCV-infected persons was associated with reduced risk for HCC (relative risk for all persons, 0.24 [95\% CI, 0.18 to 0.31 ], moderate-quality evidence; advanced liver disease hazard ratio, 0.23 [CI, 0.16 to 0.35 ], moderate-quality evidence). In contrast, Wang and Chang (2012) and Dohmen et al (2013) reported that HCC still sporadically developed even after achievement of SVR. Therefore, no data to date demonstrate that treating or eradicating hepatitis $\mathrm{C}$ could completely eliminates the risk of HCC. Thus it seems that patients with chronic HCV infection and cirrhosis who have achieved viral clearance on therapy should, at least for now, continue to undergo HCC surveillance.

Given the complexity of the clinical scenario of HCC's etiologies, in addition to $\mathrm{HBV}$ and $\mathrm{HCV}$, future work should be also focused on other HCC risk factors. Among these, aflatoxins are one of the most potent hepatocarcinogens and are easily acquired by human through exposure to mycotoxins. The incidence of HCC may be reduced by eliminating aflatoxin through proper food storage (Wogan, 1992). Several authors have reported in two meta-analyses that the relationship between coffee and HCC provided substantial evidence that there is an inverse relation between coffee and HCC (Bravi et al., 2007; Larsson and Wolk, 2007), indicating a reduced risk of liver cancer. However, the data are not conclusive. A well-designed, larger cohort of patients is still needed to validate the results of above reports. Vitamin K2 inhibits the growth of various neoplastic cells, including hepatoma cells, by causing cell-cycle arrest and apoptosis through different proposed mechanisms (Sakai et al., 1994). An ramdomized controlled trials involving the use of vitamin $\mathrm{K} 2$ in the prevention of HCC in women with HBV- or HCV-related cirrhosis proved that there could be a possible role for this as a preventive agent (Habu et al., 2004). Attention should be paid to carcinogenic effect of alcohol as well. Many authors support the fact that heavy alcohol intake is strongly associated with HCC (Donato et al., 2002). Therefore, It is reasonable to suppose that abstinence of heavy alcohol drinking is probably beneficial in reducing the risk of HCC. This will be an issue for further studies.
Nowadays, an enormous effort is being made by researchers focusing on nonalcoholic steatohepatitis (NASH), which has been reported to affect 2-3\% of the world's population, making it probably the most common liver disorder today. Of these patients with NASH, 23\% progress to liver cirrhosis in 10-15 years (Bacon et al., 1994; Matteoni et al., 1999). In this scenario, HCC development may be a part of the natural history of this disease because NASH may progress to cirrhosis (Caldwell et al., 1999; Cuadrado et al., 2005).

Current research trends with respect to obesity, which were recently and comprehensively reviewed. Epidemiological data from Chen et al. (2008) suggest that extreme obesity (body mass index $>30 \mathrm{~kg} / \mathrm{m}^{2}$ ) was independently associated with a fourfold risk of $\mathrm{HCC}$ in anti-HCV-positive subjects and a twofold risk of HCC in those without HBV or HCV after controlling for other metabolic factors. They also found more than 100-fold increased risk of $\mathrm{HCC}$ in $\mathrm{HBV}$ or HCV carriers with both diabetes and obesity, indicating synergistic effects of metabolic factors and viral hepatitis (Wang et al., 2003). Furhtermore, the correlations between liver cancer and nonalcoholic fatty liver disease (NAFLD) -related cirrhosis, and the role of the metabolic syndrome in the development of HCC from diverse etiologies, including $\mathrm{HCV}$-mediated cirrhosis are becoming increasingly recognized (Michelotti et al., 2013). Hence, control of risk factors such as type 2 diabetes, obesity, and dyslipidemia is recommended as the first and most important approach in managing people with NAFLD and NASH. Then, it is eligible for preventing development of cirrhosis and HCC. Taken together, as previously stated, a multidisciplinary approach of reduction of alcohol consumption in HBV or HCV-infected patients associated with controlling obesity and diabetes mellitus, abstinence of heavy alcohol drinking, and limiting fungal contamination of crops to reduce aflatoxin exposure that could have a real effect on diminishing HCC risk.

Tremendous efforts had been made by researchers regarding the issue of tertiary prevention of HCC recurrence after surgical resection. A nationwide cohort study (Wu et al., 2012) enrolled 100,938 newly diagnosed HCC patients. There were 4,569 HBV related HCC patients who received curative liver resection for HCC. The risk of first tumor recurrence was compared between patients not taking NAs (untreated cohort, $\mathrm{n}=4051$ ) and patients taking NAs (treated cohort, $\mathrm{n}=518$ ). They concluded that the treated cohort had a higher prevalence of liver cirrhosis when compared with the untreated cohort $(P<0.001)$, but lower risk of HCC recurrence $(P<0.001)$, and lower overall death $(P<0.001)$. Analogously, in patients of $\mathrm{HCV}$ related HCC undergoing surgical resection, Hsu et al. in their population-based research (Hsu et al., 2013) provided solid evidence that postoperative pegylated interferon plus ribavirin is associated with reduced recurrence of $\mathrm{HCC}$. They reported that the recurrence rate of $\mathrm{HCC}$ was significantly lower in the treated than untreated cohort, with $52.1 \%$ (95\% confidence interval [CI], 42.0-62.2\%) and $63.9 \%$ (95\% CI, 58.9-68.8\%) after 5 years of followup, respectively $(P=0.001)$. 


\section{Discussion}

The purpose of this review is to outline the current consunsus and discuss the latest developments in strategies of the diagnostic, therapeutic, and preventive aspects of care for patients with HCC based on innovative reports in the literature.

For decades, patients with predisposing hepatic diseases have been surveyed using optimal screening frequency of bi-annual AFP and hepatic US examination, yet accuracy for the detection rate of early stage HCC has been generally recognized to be unsatisfactory. Nevertheless, the HCC surveillance strategies are still known to be clinically beneficial and mandatory in terms of markedly different therapeutic outcomes between early $\mathrm{HCC}$, which is usually detected in regularly screened subjects, and advanced HCC, which is almost diagnosed incidentally. Only limited numbers of HCC patients at initial diagnosis are at an early stage, which enable patients to be treated with curative therapies, including liver transplantation, surgical resection, and local ablation. Unfortunately, most of the HCC patients are not candidates for these approaches. These findings provide the rationale to develop preventive strategies and surveillance plans that aims at reducing occurrence of HCC and its associated morbility and mortality.

As opposed to HCC treatment, HCC prevention consists of procedures taken before HCC development because it is a dynamic process from etiologic risk factors to $\mathrm{HCC}$, which actually begin before individuals realize they are affected. Therefore, disease prevention relies on anticipatory actions that can be categorized as primary, secondary, and tertiary prevention. Large-scale longterm follow-up studies in the literature provide ample evidence suggesting that primary prevention of HBV infection by vaccination has been effective in reducing the incidence of HCC (Hsu et al., 1988; Chen et al., 1996; Chang et al., 2000 Chang et al., 2005; Chang et al., 2009). Primary HCC prevention with universal HBV vaccination has become worldwide well-established health policy to protect children against HBV related HCC. To identify hepatotrophic virus, which have been recognized to be causally related to $\mathrm{HCC}$, is fundamental to secondary prevention of HCC development. As previously described, altrough it remains controverial, in people already infected with $\mathrm{HBV}$ or $\mathrm{HCV}$, oral anti$\mathrm{HBV}$ and pegylated interferon-based anti-HCV agents are most used therapies against $\mathrm{HCC}$ development in terms of carcinogenesis of $\mathrm{HBV}$ and $\mathrm{HCV}$ because there is abundant evidence (Morgan et al., 2013; Zoutendijk et al., 2013) that antiviral therapies with the aim to suppress $\mathrm{HBV}$ and $\mathrm{HCV}$ replication can slow disease progression or even reverse liver damage and fibrosis, eventually prevent HCC formation. Analogously, eradication of other HCC risk factors, such as alcohol consumption, obesity and diabetes mellitus, aflatoxin exposure could have a similar effect of HCC prevention. Lastly, antiviral therapies for $\mathrm{HBV}$ and HCV also play an important role in tertiary prevention of $\mathrm{HCC}$ recurrence in patients who undergo surgical resection for HCC (Wu et al., 2012; Hsu et al., 2013). The purpose of tertiary prevention is to maintain and even maximize the remaining functions of an already treated HCC patient in terms of blocking HCC recurrence and progression.

Dsepite the fact that HCC is fatal and aggressive, many innovative technologies and cutting edge methods about the management of HCC have been developed during the past decades, which have been changing from single method to multidisciplinary treatment options as well as comprehensively taking into account variables of tumor stage, underlying liver function, performance status, comorbid conditions and life expectancy. Treatment judgements of HCC are complicated and need elaborate therapeutic plans, which are dependent on tumor staging, existence or not of portal hypertension, and the extent of hepatic damage, which significantly affects the overall survival. The notions of individualized therapy of HCC suggest that the treatment strategy should be tailored on the single patient, which not only just need to be highlighted in accordance to evidence-based treatment guidelines but also highly depend on up-to-date advances. Potentially curative therapies like surgical resection, liver transplant and local ablation are not an option for most patients as they are often diagnosed when the disease is advanced. Therefore, a treatment algorithm should include both tumor- and hepatic reserve-related factors because accurate assessment and classification of disease is important for patient management.

Many novel treatment schemes have recently emerged as a therapeutic option for HCC, particularly in advanced stage. Compared with standard TACE procedure, embolization wih doxorubicin DEB may confer a durable effects and favorable outcomes (Malagari et al., 2010). As a new therapy, radioembolization (Salem et al., 2010) was developed and has now been implemented to improve outcomes and already had produced promising results. However, due to its high price and radiation exposure, the decision to initiate radioembolization therapy must weigh its benefits and risks because safety and cost are important issues to consider when dealing with this procedure. Rationale for its use needs further validation.

Sorafenib, as a first line treatment in advanced-stage HCC, was proved to improve overall survival by Llovet et al (2008) in a large prospective randomized controlled trial, and this result was again confirmed by Cheng et al (2009) in an Asian population. Since its launching into clinical use in 2008, sorafenib is currently considered a standard treatment for HCC beyond early and intermediate stage because of its potent, targeted, and tolerated characteristics. Several second line of oral targeted antiHCC agents have also been undergoing clinical trials, which are benificial in prolonging the time to progression (Bruix et al., 2013; Llovet et al., 2013; Zhu et al., 2014).

Given the fact that effective treatment of HCC is usually based on an ideal algorism of accurate diagnosis of $\mathrm{HCC}$, which is characterized by a combination of optimal imaging, laboratory, and histologic findings. AFP has gained widespread use for as a biochemical tests for HCC screening and diagnosis since 1970 s. However, its role in diagnosis is relatively limited in patients with small HCC and HCC of normal AFP level. In this scenario, as depicted in this review article, DCP, AFP-L3, and many other 
biomarkers have been accepted as better alternative tests. Dynamic CT, dynamic MRI, CTAP, and CTHA might provide a more across-the-board standardized radiological diagnosis of HCC. The issue of differentiating early HCC from dysplatic nodule (DN) used to be challenging, which had recently solved by Gd-EOB-DTPA-enhanced MRI in terms of hepatocyte-phase images (Sano et al., 2011). If there are still uncertianties about making definite diagnosis of HCC, histological features would be the last choice to solve the problems. Neverhteless, morphological characteristics alone sometimes are not evident enough to distinguish early HCC from DN. In this circumstances, thanks to the development of immunohistochemistrycross-sectional diagnostic imaging markers of early HCC identified by genomic studies, GPC3 as well as several other protein markers (Llovet et al., 2006; Wurmbach et al., 2007) may help to clarify the inconsistencies and ambiguities

\section{References}

Andea L, Isgro G, Pleguezueli M et al (2009). Surveillance and diagnosis of hepatocellular carcinoma in patients with cirrhosis. World J Hepatol, 1, 48-61.

Bacon BR, Farahvash MJ, Janney CG, Neuschwander-Tetri BA (1994). Nonalcoholic steatohepatitis: an expanded clinical entity. Gastroenterology, 107, 1103-9.

Brechot C, Gozuacik D, Murakami Y, Paterlini-Brechot P(2000). Molecular basis for the development of the hepatitis B virus (HBV)-related hepatocellular carcinoma (HCC). Semin Cancer Biol, 10, 211-31.

Burrel M, Llovet JM, Ayuso C, et al (2003). MRI angiography is superior to helical CT for detection of HCC prior to liver transplantation: an explant correlation. Hepatol, 38, 1034-2.

Bravi F, Bosetti C, Tavani A, et al (2007). Coffee drinking and hepatocellular carcinoma risk: a meta- analysis. Hepatol, 46, 430-5.

Bruix J, Sherman M (2011). American association for the study of liver diseases management of hepatocellular carcinoma: an update. Hepatol, 53, 1020-2.

Bruix J, Tak WY, Gasbarrini A, et al (2013). Regorafenib as second-line therapy for intermediate or advanced hepatocellular carcinoma: multicentre, open-label, phase II safety study. Eur J Cancer, 49, 3412-9.

Chen HL, Chang MH, Ni YH, et al (1996). Seroepidemiology of hepatitis $\mathrm{B}$ virus infection in children: ten years of mass vaccination in Taiwan. JAMA, 276, 906-8.

Chang MH, Chen CJ, Lai MS, et al (1997). For the Taiwan childhood hepatoma study group. universal hepatitis $b$ vaccination in taiwan and the incidence of hepatocellular carcinoma in Children. N Engl J Med, 336, 1855-9.

Caldwell SH, Oelsner DH, Iezzoni JC, et al (1999). Cryptogenic cirrhosis: clinical characterization and risk factors for underlying disease. Hepatol, 29, 664-9.

Chang MH, Shau WY, Chen CJ, et al (2000). Hepatitis B vaccination and hepatocellular carcinoma rates in boys and girls. JAMA, 284, 3040-2.

Capurro M, Wanless IR, Sherman M, et al (2003). Glypican-3: a novel serum and histochemical marker for hepatocellular carcinoma. Gastroenterol, 125, 89-7.

Capurro M, Wanless IR, Sherman M, et al (2003). Glypican-3: a novel serum and histochemical marker for hepatocellular carcinoma. Gastroenterol, 125, 89-97.

Chang MH, Chen TH, Hsu HM, (2005). Prevention of hepatocellular carcinoma by universal vaccination against hepatitis B virus: the effect and problems. Clin Cancer Res, 11, 7953-7.

Cuadrado A, Orive A, Garcia-Suarez C, et al (2005). Nonalcoholic steatohepatitis (NASH) and hepatocellular carcinoma. Obes Surg, 15, 442-6.

Chen CL, Yang HI, Yang WS, et al (2008). Metabolic factors and risk of hepatocellular carcinoma by chronic hepatitis B/C infection: a follow-up study in Taiwan. Gastroenterol, 135, 111-21.

Cheng AL, Kang YK, Chen Z, et al (2009). Efficacy and safety of sorafenib in patients in the Asia-Pacific region with advanced hepatocellular carcinoma: a phase III randomised, doubleblind, placebo-controlled trial. Lancet Oncol, 10, 25-34.

Chang MH, You SL, Chen CJ, et al (2009). Decreased incidence of hepatocellular carcinoma in hepatitis B vaccines: a 20year follow-up study. J Natl Cancer Inst, 101, 1348-5.

Cohn A, Kelley RK, Yang TS, et al (2012). Activity of cabozantinib (XL184) in hepatocellular carcinoma patients: results from a phase II randomized discontinuation trial (RDT), J Clin Oncol, 30, 4007.

Donato F, Tagger A, Gelatti U, et al (2002). Alcohol and hepatocellular carcinoma: the effect of lifetime intake and hepatitis virus infections in men and women. Am J Epidemiol, 155, 323-1.

Di Bisceglie AM, Sterling, RK, Chung RT, et al (2005). Serum $\alpha$-fetoprotein levels in patients with advanced hepatitis : results from the HALT-C Trial. J Hepatol, 43, 434-1.

Di Tommaso L, Franchi G, Park YN, et al (2007). Diagnostic value of HSP70, glypican 3, and glutamine synthetase in hepatocellular nodules in cirrhosis. Hepatol, 45, 725-4.

Dohmen K, Kawano A, Takahashi K, et al (2013). The incidence and risk factors for the development of hepatocellular carcinoma after peginterferon plus ribavirin therapy for chronic hepatitis C. Hepatogastroenterol, 60, 2034-8.

Dhayat SA, Mardin WA, Köhler G, et al (2014). The microRNA-200 family-A potential diagnostic marker in hepatocellular carcinoma? J Surg Oncol, in press.

El-Serag HB (2012). Epidemiology of viral hepatitis and hepatocellular carcinoma. Gastroenterology,142, 1264-3.

Franca AVC, junior, LE, Lima BLG, Martinelli ALC, Carrilho, FL (2004). Diagnosis, staging and treatment of hepatoceelualr carcinoma. Brazilian Journal of Medical and Biological Research, 37, 1689-705.

Franceschi S, Montella M, Polesel J, et al (2006). Hepatitis viruses, alcohol, and tobacco in the etiology of hepatocellular carcinoma in Italy. Cancer Epidemiol Biomarkers Prev, 15, 683-9.

Forner A, Vilana R, Ayuso C, et al (2008). Diagnosis of hepatic nodules $20 \mathrm{~mm}$ or smaller in cirrhosis: Prospective validation of the noninvasive diagnostic criteria for hepatocellular carcinoma. Hepatology, 47, 97-4.

Giannelli G, Marinosci F, Trerotoli P, et al (2005). SCCA antigen combined with $\alpha$-fetoprotein as serologic markers of HCC. Int J Cancer, 117, 506-9.

Hsu HM, Chen DS, Chuang CH, et al (1988). Efficacy of a mass hepatitis B vaccination program in Taiwan. Studies on 3464 infants of hepatitis B surface antigen-carrier mothers. JAMA, 260, 2231-5.

Habu D, Shiomi S, Tamori A, et al (2004). Role of vitamin K2 in the development of hepatocellular carcinoma in women with viral cirrhosis of the liver. JAMA, 292, 358-1.

Hsia CY, Huo TI, Chiang SY, et al (2007). Evaluation of interleukin-6, interleukin-10 and human hepatocyte growth factor as tumor markers for hepatocellular carcinoma. Eur J Surg Oncol, 33, 208-2.

Hilgard P, Hamami M, Fouly AE, et al (2010). Radioembolization 
Current Trends and Recent Advances in Diagnosis, Therapy, and Prevention of Hepatocellular Carcinoma

with yttrium-90 glass microspheres in hepatocellular carcinoma: European experience on safety and long-term survival. Hepatol, 52, 1741-9.

Hsu YC, Ho HJ, Wu MS, Lin JT, Wu CY (2013). Postoperative peg-interferon plus ribavirin is associated with reduced recurrence of hepatitis $\mathrm{C}$ virus-related hepatocellular carcinoma. Hepatol, 58, 150-7.

Hosaka T, Suzuki F, Kobayashi M, et al (2013). Long-term entecavir treatment reduces hepatocellular carcinoma incidence in patients with hepatitis B virus infection. Hepatol, 58, 98-7.

International Consensus Group for Hepatocellular Neoplasia (2009). Pathological diagnosis of early hepatocellular carcinoma: a report of the international concensus group for hepatocellular neoplasia. Hepatol, 49, 658-4.

Ichikawa T, Sano K, Morisaka H (2014). Diagnosis of Pathologically Early HCC with EOB-MRI: Experiences and Current Consensus. Liver Cancer, 3, 97-107.

Joo I, Choi BI. (2012). New paradigm for management of hepatocellular carcinoma by imaging. Liver Cancer, 1, 94-9.

Koike Y, Shiratori Y, Sato S, et al (2001). Des-gamma-carboxy prothrombin as a useful predisposing factor for the development of portal venous invasion in patients with hepatocellular carcinoma: a prospective analysis of 227 patients. Cancer, 91, 561-9.

Kudo M, Imanaka K, Chida N, et al (2011). Phase III study of sorafenib after transarterial chemoembolisation in Japanese and Korean patients with unresectable hepatocellular carcinoma. Eur J Cancer, 47, 2117-27.

Kudo M, Han G, Finn RS, et al (2014). Brivanib as adjuvant therapy to transarterial chemoembolization in patients with hepatocellular carcinoma: A randomized phase 3 trial. Hepatol, 60, 1697-7.

Llovet JM, Bru C, Bruix J (1999). Prognosis of hepatocellular carcinoma: the BCLC staging classification. Semin Liver Dis, 19, 329-8.

Llovet JM, Fuster J, Bruix J (1999). Intention-to-treat analysis of surgical treatment for early hepatocellular carcinoma: resection versus transplantation. Hepatol, 30, 1434-40.

Lim SG, Mohammed R, Yuen MF, Kao JH (2009). Prevention of hepatocellular carcinoma in hepatitis B virus infection. $J$ Gastroenterol Hepatol, 24, 1352-7.

Llovet JM, Bruix J (2003). Systematic review of randomized trials for unresectable hepatocellular carcinoma: Chemoembolization improves survival. Hepatol, 37, 429-42.

Li X, Feng GS, Zheng CS, Zhuo CK, Liu X (2004). Expression of plasma vascular endothelial growth factor in patients with hepatocellular carcinoma and effect of transcatheter arterial chemoembolization therapy on plasma vascular endothelial growth factor level. World J Gastroenterol, 10, 2878-2.

Llovet JM, Chen Y, Wurmbach E, et al (2006). A molecular signature to discriminate dysplastic nodules from early hepatocellular carcinoma in HCV cirrhosis. Gastroenterol, 31, 1758-7.

Larsson SC, Wolk A (2007). Coffee consumption and risk of liver cancer: a meta-analysis. Gastroenterology, 132, 1740-5.

Llovet JM, Ricci S, Mazzaferro V, et al (2008). SHARP Investigators Study Group Sorafenib in advanced hepatocellular carcinoma. $N$ Engl J Med, 359, 378-90.

Lok AS, Sterling RK, Everhart JE, et al (2010). Des-gammacarboxy prothrombin and alpha-fetoprotein as biomarkers for the early detection of hepatocellular carcinoma. Gastroenterol, 138, 493-2.

Lencioni R, Zou J, Leberre M, et al (2010). Sorafenib (SOR) or placebo (PL) in combination with transarterial chemoembolization (TACE) for intermediate-stage hepatocellular carcinoma (SPACE). J Clin Oncol, 28, 15.
Lok AS, Everhart JE, Wright EC, et al (2011). Maintenance peginterferon therapy and other factors associated with hepatocellular carcinoma in patients with advanced hepatitis C. Gastroenterol, 140, 840-9.

Lok AS, Gardiner DF, Lawitz E. et al (2012). Preliminary study of two antiviral agents for hepatitis C genotype 1. N Engl $J$ Med, 366, 216-4.

Lee JM, Yoon JH, Joo I, Woo HS (2012). Recent advances in CT and MR imaging for evaluation of hepatocellular carcinoma. Liver Cancer, 1, 22-40.

Lin IY, Yen CH, Liao YJ, et al (2013). Identification of FKBP11 as a biomarker for hepatocellular carcinoma. Anticancer Res, 33, 2763-9.

Llovet JM, Decaens T, Raoul JL, et al (2013). Brivanib in patients with advanced hepatocellular carcinoma who were intolerant to sorafenib or for whom sorafenib failed: results from the randomized phase III BRISK-PS study. J Clin Oncol, 31, 3509-6.

Mazzaferro V, Regalia E, Doci R, et al (1996). Liver transplantation for the treatment of small hepatocellular carcinomas in patients with cirrhosis. $N$ Engl J Med, 334, 693-9.

Matteoni CA, Younossi ZM, Gramlich T, et al (1999). Nonalcoholic fatty liver disease: a spectrum of clinical and pathological severity. Gastroenterol, 116, 1413-9.

Marrero JA, Su GL, Wei W, et al (2003). Des-gamma carboxyprothrombin can differentiate hepatocellular carcinoma from nonmalignant chronic liver disease in american patients. Hepatol, 37, 1114-1.

Marrero JA, Romano PR, Nikolaeva O, et al (2005). GP73, a resident Golgi glycoprotein, is a novel serum marker for hepatocellular carcinoma. J Hepatol, 43, 1007-2.

Mazzaferro V, Romito R, Schiavo M, et al (2006). HCC Italian task force. prevention of hepatocellular carcinoma recurrence with alpha-interferon after liver resection in $\mathrm{HCV}$ cirrhosis. Hepatol, 44, 1543-54.

Masuzaki R, Yoshida H, Omata M (2010). Does chemotherapy prevent HCV-related hepatocellular carcinoma? Pros Dig Liver Dis, 42, 281-6.

Malagari K, Pomoni M, Kelekis A, et al (2010). Prospective randomized comparison of chemoembolization with doxorubicin-eluting beads and bland embolization with BeadBlock for hepatocellular carcinoma. Cardiovasc Intervent Radiol, 33, 541-51.

Morgan RL, Baack B, Smith BD, et al (2013). Eradication of hepatitis $\mathrm{C}$ virus infection and the development of hepatocellular carcinoma: a meta-analysis of observational studies. Ann Intern Med, 158, 329-7.

Matsubara T, Kanto T, Kuroda S, et al (2013). TIE2-expressing monocytes as a diagnostic marker for hepatocellular carcinoma correlates with angiogenesis. Hepatol, 57, 1416-5.

Michelotti GA, Machado MV, Diehl AM (2013). NAFLD, NASH and liver cancer. Nat Rev Gastroenterol Hepatol, 10, 656-5.

Murakami T, Tsurusaki M (2014). Hypervascular benign and malignant liver tumors that require differentiation from hepatocellular carcinoma: key points of imaging diagnosis. Liver Cancer, 3, 85-96.

Ng KK, Vauthey JN, Pawlik TM, et al (2005). Is hepatic resection for large or multinodular hepatocellular carcinoma justified? Results from a multi-institutional database. Ann Surg Oncol, 12, 364-3.

NCCN Clinical Practical Guidances in Hepatobiliary Cancers, 2013. http://www.nccn.org/professionals/physician_gls/f_ guidelines.asp Accessed

Nakazawa T, Hidaka H, Shibuya A, et al (2014). Overall survival in response to sorafenib versus radiotherapy in unresectable 
hepatocellular carcinoma with major portal vein tumor thrombosis: propensity score analysis. BMC Gastroenterol, 14, 84-90.

Parkin DM, Bray F, Ferlay J, Pisani P (2005). Global cancer statistics, 2002. CA Cancer J Clin, 55, 74-108.

Pawlik TM, Poon RT, Abdalla EK, et al (2005). Hepatectomy for hepatocellular carcinoma with major portal or hepatic vein invasion: results of a multicenter study. Surgery, 137, 403-10.

Papatheodoridis GV, Lampertico P, Manolakopoulos S, Lok A (2010). Incidence of hepatocellular carcinoma in chronic hepatitis B patients receiving nucleos (t)ide therapy: a systematic review. J Hepatol, 53, 348-6.

Park MJ, Kim YK, Lee MW, et al (2012). Small hepatocellular carcinomas: improved sensitivity by combining gadoxetic acid-enhanced and diffusion-weighted MR imaging patterns. Radiol, 264, 761-70.

Park JW, Koh YH, Kim HB, et al (2012). Phase II study of concurrent transarterial chemoembolization and sorafenib in patients with unresectable hepatocellular carcinoma. $J$ Hepatol, 56, 1336-2.

Qi J, Wang J, Katayama H, Sen S, Liu SM (2013). Circulating microRNAs (cmiRNAs) as novel potential biomarkers for hepatocellular carcinoma. Neoplasma, 60, 135-2.

Roskams T, Kojiro M (2010). Pathology of early hepatocellular carcinoma: conventional and molecular diagnosis. Semin Liver Dis, 30, 17-25.

Sakai I, Hashimoto S, Yoda M, et al (1994). Novel role of vitamin K2: a potent inducer of differentiation of various human myeloid leukemia cell lines. Biochem Biophys Res Commun, 205, 1305-10.

Shiina S, Teratani T, Obi S, et al (2005). A randomized controlled trial of radiofrequency ablation with ethanol injection for small hepatocellular carcinoma. Gastroenterol, 129, 122-30.

Sterling RK, Jeffers L, Gordon F, et al (2007). Clinical utility of AFP-L3\% measurement in North American patients with HCV-related cirrhosis. Am J Gastroenterol, 102, 2196-5.

Singal A, Volk ML, Waljee A, Salgia R, Higgins P, et al (2009). Meta-analysis: surveillance with ultrasound for early-stage hepatocellular carcinoma in patients with cirrhosis. Aliment Pharmacol Ther, 30, 37-47.

Salem R, Lewandowski RJ, Mulcahy MF, et al (2010). Radioembolization for hepatocellular carcinoma using Yttrium-90 micro- spheres: a comprehensive report of longterm outcomes. Gastroenterology, 138, 52-64.

Sano K, Ichikawa T, Motosugi U, et al (2011). Imaging study of early hepatocellular carcinoma: usefulness of gadoxetic acid-enhanced MR imaging. Radiology, 261, 834-4.

Tsai SL, Huang GT, Yang PM, et al (1990). Plasma des-gammacarboxyprothrombin in the early stage of hepatocellular carcinoma. Hepatol, 11, 481-8.

Trevisani F, D'Intino PE, Morselli-Labate AM, et al (2001). Serum alpha-fetoprotein for diagnosis of hepatocellular carcinoma in patients with chronic liver disease: influence of HBsAg and anti-HCV status. J Hepatol, 34, 570-5.

Tateishi R, Yoshida H, Matsuyama Y, et al (2008). Diagnostic accuracy of tumor markers for hepatocellular carcinoma: a systematic review. Hepatol Int , 2, 17-30.

Triolo M, Corte CD, Colombo M (2014). Impact of HBV therapy on the incidence of hepatocellular carcinoma. Liver Int $, 34,139-5$.

Villanueva A, Minguez B, Forner A, et al (2010). Hepatocellular carcinoma: novel molecular approaches for diagnosis, prognosis, and therapy. Аnnu Rev Med, 61, 317-28.

Wogan GN (1992). Aflatoxins as risk factors for hepatocellular carcinoma in humans. Cancer Res, 52, 2114-8.

Wang RT, Koretz RL, Yee HF Jr (2003). Is weight reduction an effective therapy for nonalcoholic fatty liver? A systematic review. Am J Med, 115, 554-9.

Wilhelm SM, Carter C, Tang L, et al (2004). BAY 43-9006 exhibits broad spectrum oral antitumor activity and targets the RAF/MEK/ERK pathway and receptor tyrosine kinases involved in tumor progression and angiogenesis. Cancer Res, 64, 7099-109.

Wurmbach E, Chen YB, Khitrov G, et al (2007). Genomewide molecular profiles of $\mathrm{HCV}$-induced dysplasia and hepatocellular carcinoma. Hepatol, 45, 938-7.

Wong VW, Chan SL, Mo F, et al (2010). Clinical scoring system to predict hepatocellular carcinoma in chronic hepatitis B carriers. J Clin Oncol, 28, 1660-5.

Wang CH, Chang KH (2012). Long-term assessment of hepaticellular carcinoma riask in chronic hepatitis $\mathrm{C}$ patients with sustained viral response to antiviral treatment. Gastroenterol, 142, 1060.

Wu CY, Chen YJ, Ho HJ, et al (2012). Association between nucleoside analogues and risk of hepatitis B virus-related hepatocellular carcinoma recurrence following liver resection. JAMA, 308, 1906-4.

Wong GLH, Wong VWS (2013). Risk prediction of hepatitis B virus-related hepatocellular carcinoma in the era of antiviral therapy. World J Gastroenterol, 19, 6515-2.

Xie H, Ma H, Zhou D (2013). Plasma HULC as a Promising Novel Biomarker for the Detection of Hepatocellular Carcinoma. Biomed Res Int, 2013, 136106.

Yoshizawa H (2002). Hepatocellular carcinoma associated with hepa- titis $\mathrm{C}$ virus infection in Japan: projection to other countries in the foreseeable future. Oncol, 62, 8-17.

Yoshizawa H, Tanaka J, Miyakawa Y (2006). National prevention of hepatocellular carcinoma in Japan based on epidemiology of hepatitis $\mathrm{C}$ virus infection in the general population. Intervirology, 49, 7-17.

Yuen MF, Tanaka Y, Fong DY, et al (2009). Independent risk factors and predictive score for the development of hepatocellular carcinoma in chronic hepatitis B.J Hepatol, 50, 80-8.

Yang HI, Yuen MF, Chan HL, et al (2011). Risk estimation for hepatocellular carcinoma in chronic hepatitis B (REACH-B): development and validation of a predictive score. Lancet Oncol, 12, 568-4.

Yu NC, Chaudhari V, Raman SS, et al (2011). CT and MRI improve detection of hepatocellular carcinoma, compared with ultrasound alone, in patients with cirrhosis. Clin Gastroenterol Hepatol, 9, 161-7.

Zhu AX, Finn RS, Mulcahy M, et al (2013). A phase II and biomarker study of ramucirumab, a human monoclonal antibody targeting the VEGF receptor-2, as first-line monotherapy in patients with advanced hepatocellular cancer. Clin Cancer Res, 19, 6614-3.

Zoutendijk R, Reijnders JG, Zoulim F, et al (2013). Virological response to entecavir is associated with a better clinical outcome in chronic hepatitis B patients with cirrhosis. Gut, 62, 760-5.

Zhu AX, Kudo M, Assenat E, et al (2014). EVOLVE-1 Phase 3 study of everolimus for advanced HCC that progressed during or after sorafenib. J Clin Oncol, in press. 